\title{
Stroke Prediction Based on the Spontaneous Nystagmus Suppression Test in Dizzy Patients
}

\section{A Diagnostic Accuracy Study}

Georgios Mantokoudis, MD, Thomas Wyss, PhD, Ewa Zamaro, MD, Athanasia Korda, MD, Franca Wagner, MD, Thomas C. Sauter, MD, Hassen Kerkeni, MD, Roger Kalla, MD, Miranda Morrison, MD, and

Marco Domenico Caversaccio, MD

Neurology ${ }^{\circledR}$ 2021;97:e42-e51. doi:10.1212/WNL.0000000000012176

\section{Abstract}

\section{Objective}

Failure of fixation suppression of spontaneous nystagmus is sometimes seen in patients with vestibular strokes involving the cerebellum or brainstem; however, the accuracy of this test for the discrimination between peripheral and central causes in patients with an acute vestibular syndrome (AVS) is unknown.

\section{Methods}

Patients with AVS were screened and recruited (convenience sample) as part of a prospective cross-sectional study in the emergency department between 2015 and 2020. All patients received neuroimaging, which served as a reference standard. We recorded fixation suppression with video-oculography (VOG) for forward, right, and left gaze. The ocular fixation index $(\mathrm{OFI})$ and the spontaneous nystagmus slow velocity reduction was calculated.

\section{Results}

We screened 1,646 patients reporting dizziness in the emergency department and tested for spontaneous nystagmus in 148 patients with AVS. We analyzed 56 patients with a diagnosed acute unilateral vestibulopathy (vestibular neuritis) and 28 patients with a confirmed stroke. There was a complete nystagmus fixation suppression in $49.5 \%$ of patients with AVS, in $40 \%$ of patients with vestibular neuritis, and in $62.5 \%$ of patients with vestibular strokes. OFI scores had no predictive value for detecting strokes; however, a nystagmus reduction of less than $2 \% \mathrm{~s}$ showed a high accuracy of $76.9 \%$ (confidence interval $0.59-0.89$ ) with a sensitivity of $62.2 \%$ and specificity of $84.8 \%$ in detecting strokes.

\section{Conclusions}

The presence of fixation suppression does not rule out a central lesion. The magnitude of suppression was lower compared to patients with vestibular neuritis. The nystagmus suppression test predicts vestibular strokes accurately provided that eye movements are recorded with VOG.

\section{Classification of Evidence}

This study provides Class II evidence that in patients with an AVS, decreased fixation suppression recorded with VOG occurred more often in stroke (76.9\%) than in vestibular neuritis $(37.8 \%)$.

\author{
Correspondence \\ Dr. Mantokoudis \\ georgios.mantokoudis@ \\ insel.ch
}

\section{MORE ONLINE}

Video

\section{Class of Evidence}

Criteria for rating therapeutic and diagnostic studies

NPub.org/coe

From the Department of Otorhinolaryngology, Head and Neck Surgery (G.M., T.W., E.Z., A.K., M.M., M.D.C.), University Institute of Diagnostic and Interventional Neuroradiology (F.W.), Department of Emergency Medicine (T.C.S.), and Department of Neurology (H.K., R.K.), Inselspital, University Hospital Bern and University of Bern, Switzerland.

Go to Neurology.org/N for full disclosures. Funding information and disclosures deemed relevant by the authors, if any, are provided at the end of the article. 


\section{Glossary}

AICA = anterior inferior cerebellar artery; AUC = area under the curve; AVS = acute vestibular syndrome; CI = confidence interval; EyePos = eye position; EyeVel = eye velocity (slow phase); $\Delta$ EyeVel = nystagmus velocity reduction during fixation; FFS = failure of fixation suppression; FLAIR = fluid-attenuated inversion recovery; HINTS = head impulse, nystagmus, test of skew; $\mathbf{M R A}=$ magnetic resonance angiography; $\mathbf{O F I}=$ ocular fixation index; $\mathbf{R O C}=$ receiver operating characteristic; $\mathbf{S L}=$ slice thickness; SPV = slow phase velocity; SWI = susceptibility-weighted imaging; Tc = time constant; TOF $=$ time-of-flight; TWIST $=$ time-resolved angiography with interleaved stochastic trajectories; VOG = video-oculography.

Spontaneous nystagmus is a key clinical sign in patients with an acute vestibular syndrome (AVS). Classic teaching suggests that an increase in spontaneous nystagmus with blocked fixation, implying the presence of intact visual fixation suppression of nystagmus, implies a peripheral lesion. ${ }^{1}$ However, failure of fixation suppression (FFS) is not uniformly present in patients with vestibular strokes. ${ }^{2-4}$ Almost $50 \%$ of patients with vestibular strokes do not show focal neurologic signs except for nystagmus, which might mimic a peripheral cause of dizziness, also called pseudo-neuritis. ${ }^{5}$ Such patients are at high risk for misdiagnosis, especially when risk factors are absent (e.g., younger patients) ${ }^{6}$ or if they receive nondiagnostic CTs or MRIs within the first 24 hours of symptom onset. ${ }^{7}$ Any solution to improve diagnostic accuracy in patients with vestibular strokes is crucial. Previous studies suggested new clinical bedside tests for stroke discrimination. $^{5,8}$ The HINTS protocol ${ }^{5}$ (head impulse, nystagmus, test of skew) has higher accuracy than neuroimaging in the acute stage and searches also for nystagmus direction changes (also known as "nystagmus" test or gaze-holding test), rather than nystagmus suppression failure. ${ }^{5}$ Neurology textbooks often emphasize that failure of spontaneous nystagmus suppression (or failure of fixation suppression) points towards a central cause of dizziness. ${ }^{9}$ The accuracy of such a clinical suppression sign, however, is unknown. ${ }^{10}$

In this prospective cross-sectional study, we sought to quantify the visual suppression test of spontaneous nystagmus at the bedside by using a portable eye tracking system, also called video-oculography (VOG), and to assess its diagnostic accuracy for predicting stroke.

\section{Methods}

Patients were screened and recruited during daytime hours as part of a prospective cross-sectional study (diagnostic accuracy) in the emergency department (DETECT [Dizziness Evaluation Tool for Emergent Clinical Triage]) between July 2015 and April 2020. We included all patients older than 18 years with acute, persistent dizziness fulfilling all criteria for an AVS (new onset of continuous dizziness, nausea or vomiting, motion intolerance, spontaneous nystagmus in darkness). No further diagnostic evaluations were done before patients were enrolled in the study. We excluded patients with a history of previous dizziness, eye movement disorder, abnormal vision, or trauma or patients not fit for the examination or not able to undergo neuroimaging.
Enrolled patients received neuroimaging within 24 hours after symptom onset (acute MRI) based on clinical grounds. These MRI results were not available to the investigators at the index visit. Acute MRI sequences included axial whole brain diffusion (slice thickness [SL] $4 \mathrm{~mm}$ ), dedicated brainstem diffusion (SL $3 \mathrm{~mm}$ ), axial fluid-attenuated inversion recovery (FLAIR) (SL $4 \mathrm{~mm}$ ), axial T2-weighted imaging covering the brainstem (SL $3 \mathrm{~mm}$ ), time-of-flight (TOF) magnetic resonance angiography (MRA), susceptibility-weighted imaging (SWI) (SL $1.6 \mathrm{~mm}$ ), whole brain perfusion (SL $4 \mathrm{~mm}$ ), contrastenhanced time-resolved 3D time-resolved angiography with interleaved stochastic trajectories (TWIST) MRA, and postcontrast T1-weighted imaging (SL $4 \mathrm{~mm}$ ).

Patients with an acute unilateral vestibulopathy (vestibular neuritis) had a confirmatory neuro-otologic examination (mixed horizontal-torsional nystagmus beating away from the lesion side, no other focal neurologic signs) and a pathologic bithermal caloric test ( $>20 \%$ asymmetry) or unilateral pathologic video head impulse test $(<0.8$ vestibulo-ocular reflex gain, asymmetry $\left.>20 \%^{11}\left[1-\frac{\text { lowergain }}{\text { hishergain }} x 100\right]\right)$ based on our laboratory normative values. All patients with a diagnosed acute unilateral vestibulopathy received a delayed (3-10 days after symptom onset) MRI in order to exclude any central cause of dizziness. Patients with a suspected central vestibular syndrome and a nondiagnostic acute MRI received a second delayed MRI (3-10 days after symptom onset) as well, which served as a gold reference standard for stroke confirmation. The delayed MRI protocol included sequences with axial whole brain diffusion (SL $4 \mathrm{~mm}$ ), dedicated brainstem diffusion (SL $3 \mathrm{~mm}$ ), axial T2-weighted imaging covering the brainstem (SL $3 \mathrm{~mm}$ ), TOF MRA, SWI (SL $1.6 \mathrm{~mm}$ ), native T1-weighted imaging (SL $4 \mathrm{~mm}$ ), and coronal FLAIR (SL $4 \mathrm{~mm}$ ). In suspicion of some no vessel pathology contrast medium was applied and an additional contrast-enhanced time-resolved 3D TWIST-MRA and postcontrast T1weighted imaging (SL $4 \mathrm{~mm}$ ) were acquired.

All MRIs were assessed by a neuroradiologist on duty. A second, board-certified neuroradiologist, blinded to the patient records, reviewed all images. Discrepancies were resolved by consensus and interrater concordance reported. All patients received a clinical follow-up 30 and 90 days after symptom onset.

An experienced neuro-otologist recorded nystagmus within 2-72 hours after symptom onset in the emergency 
department (bedside) or in the vestibular laboratory (when patients were mobile) using an eye tracker (EyeSeeCam) with an infrared $250 \mathrm{~Hz}$ USB camera. Tests, whether performed in the emergency department or in the laboratory, were identical. VOG device was calibrated by projecting dots (6.17 Lux, $4 \mathrm{~mm}$ diameter, visual angle size of $0.8814^{\circ}$ ) on a television screen or a tablet with a predefined distance using a chin rest $(550 \mathrm{~mm}$ distance $)$ or a small bucket $(260 \mathrm{~mm}$ distance; online-only data supplement, video). Visual fixation was removed by testing in darkness. The patients looked in different gaze directions following flashing lights $(750 \mathrm{~ms}$ visible, 2,500 ms dark period, ${ }^{12} 6.17$ Lux luminosity, $4 \mathrm{~mm}$ diameter dot, visual angle size of $0.8814^{\circ}$ ) displayed on a television screen or tablet at the bedside. The goal of the flashing light was to draw the eye to the appropriate target at $0^{\circ}\left(+/-5^{\circ}\right)$ and $\pm 7^{\circ}$ (right/left gaze) and to record slow phase velocity (SPV) during the interval of the extinguished light. The protocol for visual fixation included permanent target lights (6.17 Lux luminosity, $4 \mathrm{~mm}$ diameter dot, visual angle size of $\left.0.8814^{\circ}\right)$ at center position and $7^{\circ}$ eccentric positions.

Nystagmus SPV for each gaze direction was calculated by differentiating positional signals and removing fast phases (de-saccading) using MATLAB (MATLAB R2019b, MathWorks) script. We analyzed nystagmus velocity for all measured gaze directions but segregated the results for looking in the direction of the slow or the fast phase. We did not analyze data at tested gaze directions if there was no nystagmus or if gaze position was inaccurate (being out of range) in darkness. We described the degree of fixation suppression by calculating the difference of slow phase eye velocity with and without visual fixation ( $\Delta$ EyeVel) at each gaze direction (EyePos) separately. The ocular fixation index (OFI score [\%]) was calculated as follows: OFI $[\%]=\frac{\text { EyeVelwithfixation }}{\text { EyeVelwithoutfixation }} * 100$. The time constant $(\mathrm{Tc})$ as a measure of the neural integrator integrity can be calculated with $\mathrm{Tc}=\frac{\Delta \text { EyePos }}{\Delta \text { EyeVelGaze }}$, taking into account that $\Delta$ EyePos $=$ EyePos (right gaze) - EyePos (left gaze) and $\Delta$ EyeVelGaze $=$ EyeVel (right gaze $)-$ EyeVel (left gaze). We fitted a linear regression line through the positional vs velocity data plot with tau $=$ slope of the curve and $\mathrm{T} c=\frac{1}{\text { tau }}$.

We chose a linear regression through data points instead of a sigmoid curve in order to fit moderate eccentric data points $\left(7-15^{\circ}\right)$ and to avoid a bias by the nonlinearity of the behavior of the neural integrator especially for extremely eccentric gaze positions $\left(>20^{\circ}\right){ }^{13}$

\section{Statistics}

For the comparison of nystagmus intensity between central and peripheral disorders, we only included patients with a confirmed stroke or vestibular neuritis. We reported Cohen $\kappa$ for interrater agreement between 2 neuroradiologists. A nonparametric test (Mann-Whitney $U$ test) was applied for several gaze positions (eccentric and primary horizontal eye position), with and without visual fixation. We compared relative differences of spontaneous nystagmus velocity
( $\Delta$ EyeVel), the ratio of spontaneous nystagmus (OFI score), and differences regarding the time between symptom onset and recording. A Bonferroni correction for multiple testing with a significance level of $p=0.05$ was applied. A correlation analysis was performed (Pearson) to assess any positive relationship between nystagmus intensity and duration of dizziness at testing time.

A binary logistic regression was used for assessing the predictive measures regarding a vestibular stroke. Sensitivity and specificity was calculated using a receiver operating characteristic (ROC) curve. The cutoff for best discrimination was chosen based on the Jouden J. We followed the Standards for Reporting of Diagnostic Accuracy (STARD) guidelines. Our estimated sample size was 52 with an estimated marginal error of 0.1 (95\% confidence interval [CI]) and a diagnostic accuracy (area under the curve [AUC]) of 0.75 . We used SPSS statistical software (IBM SPSS Statistics for Windows, version 25.0) for the analysis.

\section{Standard Protocol Approvals, Registrations, and Patient Consents}

The institutional review board approved this study (KEK \#047/14; approval date 04/24/2014). All patients gave written informed consent.

\section{Data Availability}

A minimal dataset is available on request to the corresponding author.

\section{Results}

We screened for spontaneous nystagmus in 1,646 dizzy patients, included 148 patients with AVS between 20 and 91 years of age (mean $55.8 \pm$ SD 15.3 years), and present 56 patients with a diagnosed vestibular neuritis (mean \pm SD age $53.4 \pm 16.2$ years, 26 female, 30 male) and 28 patients with a confirmed stroke (mean \pm SD age $63.8 \pm 13.4$ years, 10 female, 18 male). Vascular territories included the posterior inferior cerebellar artery (17), superior cerebellar artery (3), anterior inferior cerebellar artery (AICA) (2), basilar artery (3), vertebral artery (2), anterior cerebral artery (1), and middle cerebral artery (4). Some of the patients had multiple vascular territories involved. There was excellent interrater agreement regarding masked MRI assessment $(\kappa=0.892, p<$ 0.001 ). A total of 6 patients had spontaneous nystagmus that abated within 48 hours. These patients were excluded from the analysis because patients with episodic vertigo did not fulfill all diagnostic criteria for a definitive diagnosis. Figure 1 shows a flowchart regarding patient screening, inclusions, and exclusions. We excluded 32 patients with no or unclear diagnosis. These dizzy patients had either a normal vestibular function but a nondiagnostic MRI or symptoms abated within 48 hours after onset (figure 1). All included patients tolerated VOG recordings performed between 2 and 72 hours after symptom onset (mean \pm SD $33 \pm 20 \mathrm{~h}$ ). 
Prevalence of Nystagmus Fixation Suppression There was a complete nystagmus fixation suppression (no nystagmus in light) in $49.5 \%$ of all included patients with AVS, in $40 \%$ of patients with vestibular neuritis, and in $62.5 \%$ of patients with vestibular strokes. Thus, there was a large proportion of patients with a peripheral vestibular syndrome (60\%) who failed to fully suppress their nystagmus. All patients with stroke were able to suppress at least partially their nystagmus under visual fixation. Seven out of 20 patients with stroke reduced their nystagmus velocity $(\Delta \mathrm{EyeVel})$ less than $1 \%$; however, we did not identify any stroke location clustering in these patients. A video example for VOG (setup and eye movements in patients with vestibular neuritis or stroke) is shown in the video.

\section{Nystagmus Intensity in Strokes and Vestibular Neuritis}

Table 1 and figure 2, A and B show means of nystagmus velocity (EyeVel) for each gaze position with and without visual fixation and each diagnostic group. Overall, mean eye velocity (SPV) in any gaze direction and under all conditions was significantly higher in patients with peripheral causes (figure $2 \mathrm{~A}$ ) compared to patients with vestibular strokes (table 1 and figure $2 \mathrm{~B}$ ). The duration of symptoms at testing time was not statistically different $(p=0.32)$ between cohorts. There was also no significant correlation between nystagmus intensity and duration of symptoms or between the suppression effect and duration of symptoms for both studied groups. Regarding vestibular strokes, we recorded a maximal velocity of $13.4^{\circ} \mathrm{s}$ in darkness and $3.0^{\circ} \%$ under visual fixation (compared to vestibular neuritis with a maximum EyeVel of $22.5 \%$ s and $17.7 \%$ s correspondingly).

\section{OFI and Its Accuracy}

There was statistically no difference when comparing the OFI scores between peripheral and central causes (table 1). OFI scores had no predictive value for detecting strokes in any gaze direction. The prediction of strokes using OFI scores in

Figure 1 Flow Diagram Shows the Process of Screening, Inclusions, Exclusions, and Enrollment

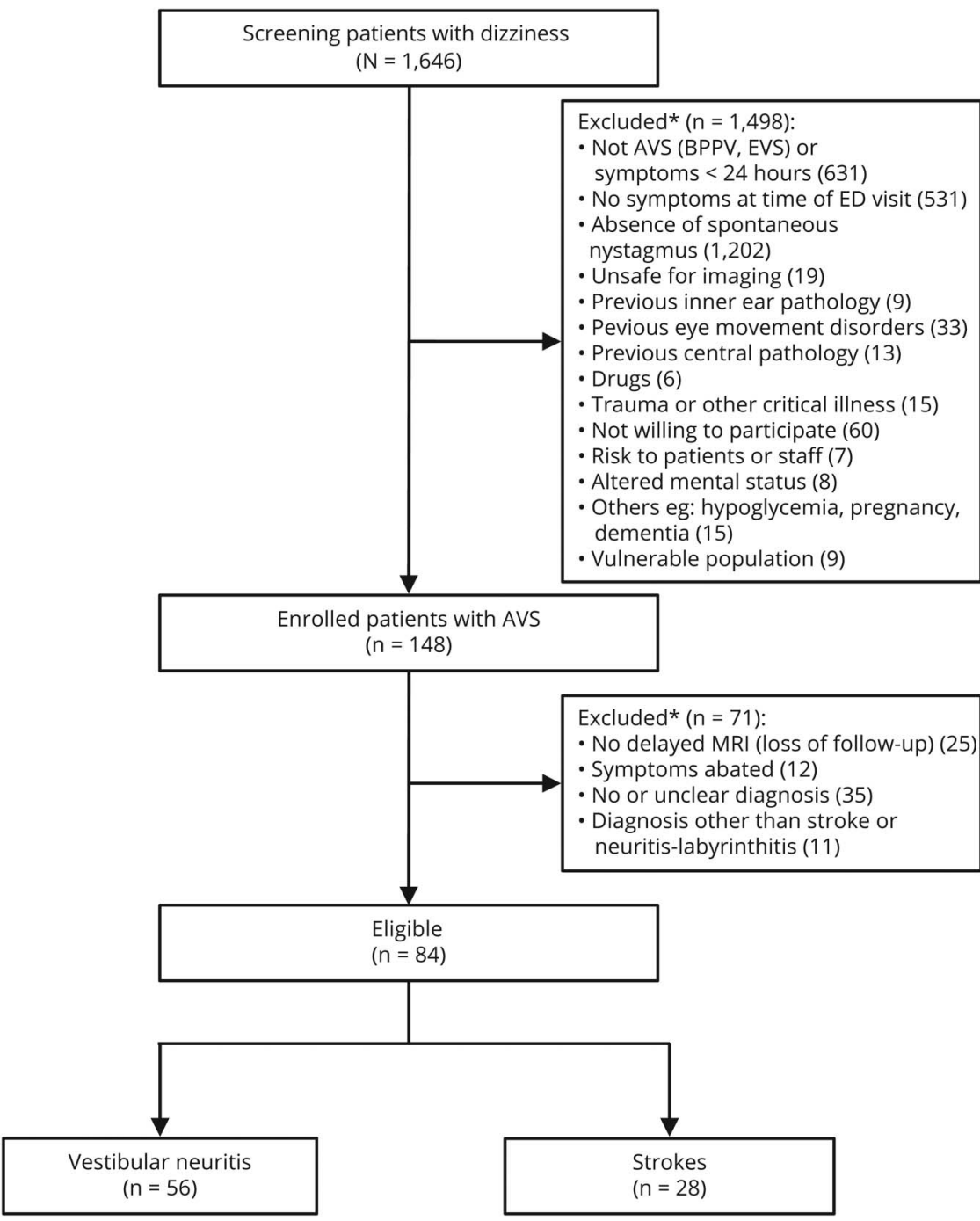

*Patients could have had one or several reasons for exclusion. AVS = acute vestibular syndrome; $\mathrm{BPPV}=$ benign paroxysmal positional vertigo; $\mathrm{ED}=$ emergency department; EVS = episodic vestibular syndrome. 
eccentric gaze positions, however, was lower than chance (AUC 0.462, $p=0.657$, CI 0.29-0.63). The calculated time constant was not predictive either (odds ratio $1, p=0.6, \mathrm{CI}$ 0.99-1.001).

\section{Nystagmus Velocity Reduction by Visual Fixation}

Looking at the nystagmus velocity reduction during fixation ( $\Delta$ EyeVel; table 1 , figure $2 \mathrm{C}$ ), we observed a statistical difference between strokes and no strokes in all gaze directions. Table 2 shows the odds ratio ranging from 0.65 to 0.74 depending on gaze direction with a significant reduction of stroke probability for each increment of $1 \%$ s EyeVel (primary gaze, odds ratio $0.647, p=0.014$, CI $0.457-0.917$ ). The best discrimination test for stroke proved to be in gaze direction of $7^{\circ}$ towards the fast phase of nystagmus velocity: figure 3 shows an ROC curve going to the left upper corner with a sensitivity of $62.2 \%$ and specificity of $84.8 \%$. Table 3 shows an overview of the sensitivity and specificity of all tested measures. Fixation suppression failure with a threshold of less than $\sim 2 \%$ s nystagmus reduction $(\Delta$ EyeVel $)$ was predicting stroke and discriminating best between strokes and vestibular neuritis (AUC 0.74, $p=0.005$, CI 0.59-0.89). Overall test accuracy was $76.9 \%$. The test sensitivity increased from $63.2 \%$ to $78.9 \%$ when looking $7^{\circ}$ in the direction of the slow phase while sacrificing specificity (72.7\%). A negative likelihood ratio of 0.434 means a reduction of posttest probability of stroke between 5.4 and 19.7 percent points if the pretest probability ranges between $10 \%$ and $75 \%$ (table 4 ). A positive likelihood of 4.168 means, however, an approximate increase in stroke posttest probability ranging between $21.6 \%$ and $33.2 \%$ points.

\section{Clinical HINTS and the Nystagmus Suppression Test}

All patients with stroke had an NIH Stroke Scale score $\leq 4$. Fourteen out of 28 patients (50\%) had no focal neurologic signs and would not have been detected based on a general emergency department or neurologic examination. Eleven patients with stroke had a vertical nystagmus component in addition to the horizontal nystagmus. Two patients had a predominantly vertical nystagmus: one was fully suppressed by fixation and the other had an incomplete suppression (OFI 56\%).

Table 1 Mean Slow Phase Velocity of Spontaneous Nystagmus Stratified by Diagnosis

\begin{tabular}{|c|c|c|c|c|c|c|c|c|}
\hline \multirow[b]{2}{*}{ Parameter/gaze direction } & \multicolumn{3}{|c|}{ Vestibular neuritis } & \multicolumn{3}{|c|}{ Stroke } & \multirow[b]{2}{*}{$p$ Value } & \multirow[b]{2}{*}{ Corrected $p$ value ${ }^{a}$} \\
\hline & $\mathbf{N}$ & Mean & $\mathrm{Cl}$ & $\mathbf{N}$ & Mean & $\mathrm{Cl}$ & & \\
\hline Age, y & 56 & 53.4 & 4.3 & 28 & 63.8 & 4.9 & & \\
\hline \multicolumn{9}{|l|}{ In room light (visual fixation) } \\
\hline Time constant & 38 & 347.6 & 537.7 & 23 & 107.6 & 56.9 & 0.341 & 1.023 \\
\hline $7^{\circ}-15^{\circ}$ fast phase ${ }^{b}$ & 35 & 2.5 & 1.2 & 24 & 0.9 & 0.4 & 0.021 & 0.063 \\
\hline Primary gaze & 39 & 1.8 & 0.7 & 25 & 0.6 & 0.3 & 0.001 & 0.003 \\
\hline $7^{\circ}-15^{\circ}$ slow phase ${ }^{c}$ & 35 & 1.7 & 1.0 & 24 & 0.5 & 0.3 & 0.018 & 0.054 \\
\hline \multicolumn{9}{|c|}{ In darkness (no visual fixation) } \\
\hline $7^{\circ}-15^{\circ}$ fast phase & 41 & 7.8 & 1.5 & 20 & 3.4 & 1.5 & $<0.001$ & $<0.001$ \\
\hline Primary gaze & 48 & 6.2 & 1.5 & 25 & 1.7 & 0.8 & $<0.001$ & $<0.001$ \\
\hline $7^{\circ}-15^{\circ}$ slow phase & 41 & 5.0 & 1.4 & 20 & 1.9 & 1.0 & 0.005 & 0.015 \\
\hline \multicolumn{9}{|l|}{ Ocular fixation index } \\
\hline $7^{\circ}-15^{\circ}$ fast phase & 32 & 0.4 & 0.3 & 18 & 0.4 & 0.2 & 0.657 & 1.971 \\
\hline Primary gaze & 38 & 0.4 & 0.1 & 22 & 0.4 & 0.2 & 0.976 & 2.928 \\
\hline $7^{\circ}-15^{\circ}$ slow phase & 32 & 0.6 & 0.3 & 18 & 0.4 & 0.3 & 0.312 & 0.936 \\
\hline \multicolumn{9}{|l|}{$\Delta$ EyeVel ( $\% / s)$} \\
\hline $7^{\circ}-15^{\circ}$ fast phase & 33 & 4.7 & 1.1 & 20 & 2.6 & 1.3 & 0.003 & 0.009 \\
\hline Primary gaze & 39 & 3.5 & 0.9 & 25 & 1.3 & 0.6 & $<0.001$ & $<0.001$ \\
\hline $7^{\circ}-15^{\circ}$ slow phase & 33 & 3.3 & 0.9 & 20 & 1.4 & 0.8 & 0.003 & 0.009 \\
\hline
\end{tabular}

Abbreviation: $\mathrm{Cl}$ = confidence interval.

${ }^{a}$ Corrected for multiple testing.

${ }^{b}$ Fast phase direction = gaze direction towards the healthy side.

c Slow phase direction = gaze direction towards the affected side. 
Figure 2 Box Plots Demonstrate Lower Quartile, Median, and Upper Quartile of Nystagmus Slow Phase Velocity (SPV) for Patients With Vestibular Neuritis and Stroke

A. Vestibular neuritis

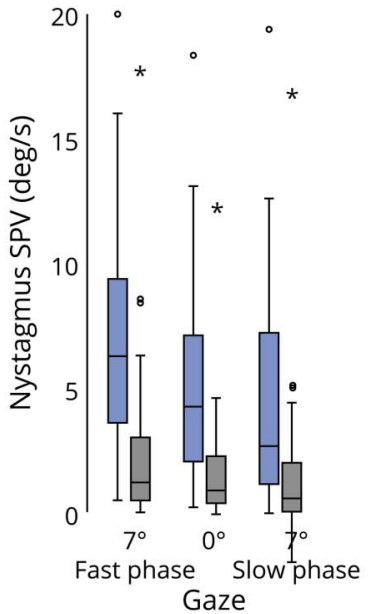

B. Stroke

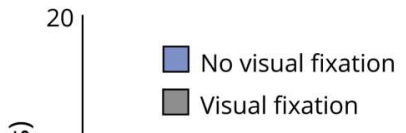

C. Suppression test

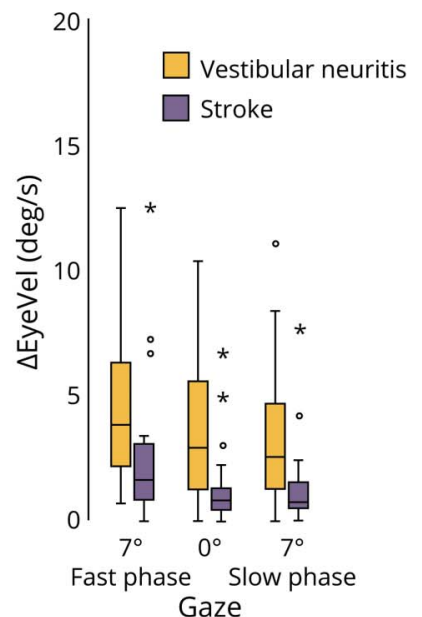

(A) Vestibular neuritis. (B) Stroke. Whiskers represent 1.5 times the interquartile range $(0=$ outliers $<1.5$ box size distance, * = outliers $>3$ box size distance). Data are grouped by gaze direction. $0^{\circ}=$ gaze straight ahead. The direction of the nystagmus beats corresponds to the fast phase direction. Gray boxes indicate measurements with visual fixation, blue boxes without visual fixation. (C) Median reduction of nystagmus velocity ( $\Delta$ EyeVel), with visual fixation. Green boxes represent patients with vestibular neuritis; red boxes, patients with stroke.
The subanalysis of clinical HINTS in our patient sample ( $\mathrm{n}=63 ; 27$ strokes and 36 vestibular neuritis) showed a sensitivity of $81.5 \%$ and $75 \%$ specificity. When combining clinical HINTS with the nystagmus suppression test, sensitivity increased to $88.9 \%$ and specificity was $69.4 \%$. Two out of 27 strokes had a false HINTS examination (peripheral type) but a true pathologic (central) nystagmus suppression test. In one of these 2 patients there was a left-sided acute infarct of the posterolateral medulla oblongata. The other patient showed an extensive infarct demarcation of the left cerebellar hemisphere with involvement of the left caudal cerebellar lobe, the left flocculus, the left posterior medulla oblongata, and the inferior vermis.

\section{Discussion}

Quantitative recording of nystagmus velocity using a portable VOG device was feasible and gave a quantitative estimate of nystagmus suppression while fixating a light target. We were able to accurately predict stroke with a moderate sensitivity but high specificity when testing patients at eccentric eye positions of $7^{\circ}$ towards the direction of nystagmus beats (fast phase); however, testing in primary eye position (looking straight forward) was less accurate. In addition, patients with stroke often had a lower nystagmus intensity (EyeVel) than patients with vestibular neuritis.

A recent study including cerebellar strokes showed a central nystagmus intensity of the horizontal component of spontaneous nystagmus ranging from 1 to $13^{\circ} / \mathrm{s}\left(4.7 \pm 3.4^{\circ} / \mathrm{s}\right){ }^{2}$ which is exactly in line with our findings. High nystagmus intensity (slow phase velocity of $>13^{\circ} / \mathrm{s}$ ) may help to rule out stroke in AVS, although studies of more patients with AICA territory infarction are needed to confirm that labyrinthine involvement does not produce high-velocity nystagmus. There is still a large diagnostic overlap because peripheral nystagmus ranged from almost 0 to $22.5^{\circ}$ s depending on the severity of semicircular canal paresis and its vestibular tone imbalance. In addition, nystagmus goggles are necessary for the visualization of suppressed nystagmus by removal of visual suppression. ${ }^{14}$

OFI scores proved to be variable and were not a reliable diagnostic predictor for stroke. A high variability was also

Table 2 Logistic Regression and Predictive Variables

\begin{tabular}{|c|c|c|c|c|c|c|c|c|c|}
\hline \multirow[b]{2}{*}{$\Delta$ EyeVel, gaze direction } & \multirow[b]{2}{*}{$\mathbf{N}$} & \multirow[b]{2}{*}{ Regression coefficient } & \multirow[b]{2}{*}{ Standard error } & \multirow[b]{2}{*}{ Wald } & \multirow[b]{2}{*}{$d f$} & \multirow[b]{2}{*}{$p$ Value } & \multirow[b]{2}{*}{ Odds ratio } & \multicolumn{2}{|l|}{$95 \% \mathrm{Cl}$} \\
\hline & & & & & & & & Lower limit & Upper limit \\
\hline $7^{\circ}-15^{\circ}$ fast phase & 52 & -0.240 & 0.118 & 4.142 & 1 & 0.042 & 0.786 & 0.624 & 0.991 \\
\hline Primary gaze & 52 & -0.435 & 0.178 & 5.987 & 1 & 0.014 & 0.647 & 0.457 & 0.917 \\
\hline $7^{\circ}-15^{\circ}$ slow phase & 52 & -0.412 & 0.180 & 5.244 & 1 & 0.022 & 0.662 & 0.465 & 0.942 \\
\hline
\end{tabular}

Abbreviations: $\mathrm{Cl}=$ confidence interval; $\Delta$ EyeVel = the difference of nystagmus velocity for recordings in light and in darkness. 
Figure 3 Receiver Operating Characteristic Curve for the Nystagmus Suppression Test in Different Gaze Positions for Differentiating Dizzy Patients With and Without Stroke

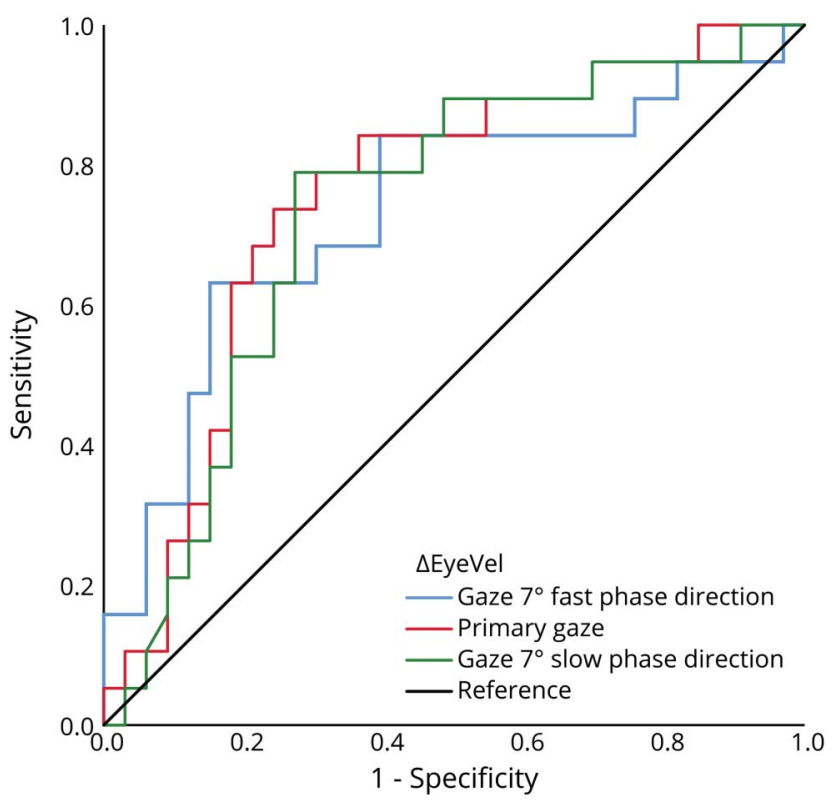

$\Delta$ EyeVel represents any eye velocity (slow phase velocity) difference between testing in darkness and in light. The diagonal reference line represents a likelihood ratio of 1 at all thresholds, indicating a useless test. The best discrimination cutoff (best sensitivity and specificity) for stroke is indicated for each test condition.

reported for the fixation suppression index of caloric-induced nystagmus. ${ }^{15}$ One potential problem with OFI scores is the problem of dividing values through zero or values close to zero explaining this variability. OFI scores are derived from a normalization procedure and represent a relative proportion of nystagmus suppression. We therefore do not recommend using OFI scores as they offer little diagnostic value.

Numerous prior studies have shown that patients with central lesions (e.g., tumors, degenerative diseases, strokes) have partial or complete FFS of either spontaneous or caloricinduced nystagmus. ${ }^{2,16-19}$ Some recent studies have examined this issue in patients with acute lesions, ${ }^{2-4}$ but, to our knowledge, none has reported quantitative criteria for distinguishing central and peripheral pathologies based on FFS characteristics. FFS is predominately seen in cerebellar patients with floccular or nodular lesions ${ }^{2,20}$ including projections to the brainstem. In addition, smooth pursuit impairment is often correlated with impaired fixation suppression of vestibular nystagmus. ${ }^{21}$ Patients with lesions affecting the extensive smooth pursuit pathways (frontal eye field, midbrain, temporal region, pons, medulla, vestibulo cerebellum) might therefore develop FFS of vestibular nystagmus. We did not find, however, a specific clustering of stroke locations in patients with FFS considering the wide range of neuronal networks involved in fixation suppression.
Nystagmus velocity reduction $(\Delta \mathrm{EyeVel})$ of less than $\sim 2 \%$ showed a high accuracy of $76.9 \%$ in detecting stroke; however, this holds true only for decent eccentric gaze positions and not for the primary gaze position. There are several reasons for this finding. Nystagmus intensity is reduced under fixation and an initially low-intensity second-degree nystagmus in darkness might become a first-degree nystagmus in light, which is only seen in gaze direction of the fast phase. A too far eccentric gaze, however, might interact with the gaze holding mechanism, which is a reason why we tested only at decent eccentric $\left(7^{\circ}-15^{\circ}\right)$ gaze. The physiologic eye drift toward the center might enhance or reduce nystagmus intensity at a far end gaze. Finally, a gaze-holding nystagmus, seen with central vestibular lesions, might not show any nystagmus at primary position.

Our study offers for the first time a VOG quantification of nystagmus intensity in patients with AVS and its diagnostic value. This clinical test (without VOG) is challenging because the reduction of nystagmus velocity (including amplitude and frequency) under visual fixation might be variable. Some of the patients might predominately reduce their frequency, others the amplitude of nystagmus beats and thus reducing their overall SPV of nystagmus. VOG systems are far more sensitive in detection of nystagmus compared to a physician's eye. $^{22}$ The diagnostic accuracy of a physician might therefore markedly differ from the diagnostic accuracy of a VOG device. The difference between experts, nonexperts, and a VOG device has not been studied. Although there is strong evidence that experts in the vestibular field reach high diagnostic accuracy applying the clinical HINTS protocol in acutely dizzy patients, ${ }^{5}$ these results might not be generalizable to a stroke specialist, a general neurologist, or an emergency physician. VOG devices might therefore offer a new way of standardizing this clinical test regardless of the skills of the testing physician. $^{11,23}$

Different viewing conditions (television vs tablet) and the presence of a flashing target light in darkness might influence ${ }^{24}$ or even induce a fixation suppression effect because patients can induce fixation suppression to an imagined or remembered/afterimage target. ${ }^{25}$ However, we reduced such effects by using a standardized protocol ${ }^{12}$ and by keeping the measures as constant as possible. The prevalence of the selected diagnostic groups might have been biased due to the chosen spectrum of patients (inclusion of 2 AICA infarctions and referral bias as a tertiary referral center) and the exclusion of patients with uncertain diagnosis. ${ }^{26}$ Patients with a nondiagnostic MRI and short duration of symptoms ( $<24$ hours) might have had TIA, vestibular migraine, or Ménière disease. The analysis of nystagmus was limited to patients who had nystagmus at the predefined gaze directions, met the recording quality criteria, and were able to follow the protocol. Thus, a comparison between different gaze positions is limited due to selection bias. The number of included patients with AVS was small. Further large-scaled trials are needed to validate our findings in patients with AVS. 
Table 3 Receiver Operating Characteristic Curves

\begin{tabular}{|c|c|c|c|}
\hline \multirow[b]{2}{*}{ Gaze direction } & \multicolumn{3}{|c|}{ Nystagmus suppression test } \\
\hline & $7^{\circ}-15^{\circ}$ fast phase & Primary $\left(0^{\circ}\right)$ position & $7^{\circ}-15^{\circ}$ slow phase \\
\hline Area under the curve & 0.735 & 0.754 & 0.733 \\
\hline SE & 0.076 & 0.070 & 0.072 \\
\hline$p$ Value & 0.005 & 0.002 & 0.006 \\
\hline 95\% Lower limit & 0.585 & 0.617 & 0.591 \\
\hline 95\% Upper limit & 0.885 & 0.892 & 0.875 \\
\hline Positive, if smaller or equal ${ }^{a}(\Delta$ EyeVel) & $1.915 \% / \mathrm{s}$ & $1.2 \% \mathrm{~s}$ & $1.72 \% \mathrm{~s}$ \\
\hline Sensitivity & $63.2 \%$ & $73.7 \%$ & $78.9 \%$ \\
\hline Specificity & $84.8 \%$ & $75.8 \%$ & $72.7 \%$ \\
\hline Positives & 17 & 22 & 24 \\
\hline Negatives & 35 & 30 & 28 \\
\hline True positive & 12 & 14 & 15 \\
\hline False positive & 5 & 8 & 9 \\
\hline True negative & 28 & 25 & 24 \\
\hline False negative & 7 & 5 & 4 \\
\hline Accuracy & $76.9 \%$ & $75 \%$ & $75 \%$ \\
\hline Likelihood ratio positive test & 4.168 & 3.039 & 2.895 \\
\hline Likelihood ratio negative test & 0.434 & 0.347 & 0.289 \\
\hline
\end{tabular}

Abbreviation: $\Delta$ EyeVel $=$ the difference of nystagmus velocity for recordings in light and in darkness.

${ }^{\text {a }}$ Cutoff.

The HINTS examination includes the test of nystagmus searching for nystagmus direction changes (gaze-holding nystagmus or pathologic gaze-evoked nystagmus) rather than failure of nystagmus suppression. Such clinical tests, however, need a clinical expertise that may not be available in smaller community hospitals. The idea of an "ECG of the eyes" ${ }^{11}$ for testing in the emergency department is a quantitative method of eye position recording in analogy to electrical potential recording in ECG. The use of VOG enables physicians to quantify vestibular reflexes while moving the head rapidly from side to side. ${ }^{27}$ These rapid, impulse-like head movements are the first part of the HINTS protocol (head impulse

Table 4 Pretest and Posttest Probabilities of Stroke Using Video-Oculography Nystagmus Suppression Tests to Rule Out or Rule In Stroke

\begin{tabular}{|c|c|c|}
\hline \multirow[b]{2}{*}{ Test } & \multicolumn{2}{|l|}{ Posttest probability of stroke } \\
\hline & $\begin{array}{l}\text { Normal nystagmus suppression test (rule out } \\
\text { stroke), gaze fast phase }\end{array}$ & $\begin{array}{l}\text { Nystagmus suppression test failure (rule in } \\
\text { stroke), gaze fast phase }\end{array}$ \\
\hline \multirow{2}{*}{$\begin{array}{l}\text { Pretest probability of stroke (based on risk } \\
\text { stratification rules) }\end{array}$} & Sensitivity $63.2 \%$, specificity $84.8 \%$ & \\
\hline & LR- 0.434 & $L R+4.168$ \\
\hline $10 \%$ (low) & 4.6 & 31.6 \\
\hline $25 \%$ (average) & 12.6 & 58.2 \\
\hline $50 \%$ (high) & 30.3 & 80.7 \\
\hline 75\% (very high) & 56.6 & 92.6 \\
\hline
\end{tabular}

Abbreviation: $L R=$ likelihood ratio.

Values are percentages. 
test), showing a VOG accuracy of $88 \%$ in detecting vestibular strokes. ${ }^{23}$ However, VOG has never been used systematically for the assessment of the other 2 measures of HINTS. An extended HINTS protocol could not only test for nystagmus direction but also measure the ability of nystagmus suppression because this is not considered as an isolated, standalone test. Commercial VOG devices offering nystagmus quantification (videonystagmography) cost between $\$ 4,000$ and $\$ 12,000$ USD and can also be used by nonexperts/novices. ${ }^{28}$ Some of the devices use a laser source mounted on the goggles frame in order to project targets on the opposite wall of a sitting patient; other devices use a semi-standardized method using the finger of the examiner as a target while recording the actual eye position. Care should be taken when interpreting the results. We recommend always looking at the recorded nystagmus curves (positional data plot) to identify possible artifacts such as eye blinks, low-quality eye tracking, or patient noncompliance. ${ }^{29}$ The impact of artifacts has been studied for VOG in previous studies, but algorithms calculating eye velocity have been found robust in terms of diagnostic accuracy. ${ }^{29,30}$

VOG devices might be used in the future to quantify all 3 steps of the HINTS protocol. Adding one more measure to a VOG HINTS protocol might further improve its diagnostic accuracy, which has to be assessed in future studies. In general, the HINTS battery works best in terms of sensitivity if applied in patients with acute and sustained dizziness rather than those with episodic or chronic vertigo. The presence of spontaneous nystagmus serves as an objective clinical sign or "biomarker" for the underlying vestibular imbalance; however, the sensitivity of HINTS in detecting stroke is not known for dizzy patients without spontaneous nystagmus.

The presence of fixation suppression does not rule out a central lesion. The magnitude of suppression $(\Delta \mathrm{EyeVel})$ was lower compared to patients with acute unilateral vestibulopathy. The nystagmus suppression test still predicts accurately vestibular strokes provided that eye movements are recorded with VOG. We suggest therefore adding one more quantitative measure to the HINTS examination. This new approach involving VOG devices in the emergency department might pave the way for a fast and cost-effective diagnosis of vestibular strokes.

\section{Study Funding}

Eyeseetec company loaned the VOG goggles. This study was supported by the Swiss National Science Foundation \#320030_173081.

\section{Disclosure}

None of the investigators has any relevant financial interests, activities, relationships, or affiliations that represent a relevant financial conflict of interest with respect to the conduct or analysis of this study. Go to Neurology.org/ $\mathrm{N}$ for full disclosures.

\section{Publication History}

Received by Neurology December 4, 2020. Accepted in final form March 26, 2021.

\section{Appendix Authors}

\begin{tabular}{lll}
\hline Name & Location & Contribution \\
\hline $\begin{array}{l}\text { Georgios } \\
\text { Mantokoudis, } \\
\text { MD }\end{array}$ & $\begin{array}{l}\text { Department of } \\
\text { Otorhinolaryngology, Head } \\
\text { and Neck Surgery, } \\
\text { Inselspital, University } \\
\text { Hospital Bern and University } \\
\text { of Bern, Switzerland }\end{array}$ & $\begin{array}{l}\text { Conceptualization, } \\
\text { methodology, data analysis, } \\
\text { drafting the article, } \\
\text { supervision, project } \\
\text { administration, funding } \\
\text { acquisition }\end{array}$ \\
\hline
\end{tabular}

Thomas Wyss, Department of Software, data analysis,

PhD Otorhinolaryngology, Head critical revision of the article, and Neck Surgery, final approval of the version Inselspital, University to be published Hospital Bern and University of Bern, Switzerland

\begin{tabular}{|c|c|c|}
\hline $\begin{array}{l}\text { Ewa Zamaro, } \\
\text { MD }\end{array}$ & $\begin{array}{l}\text { Department of } \\
\text { Otorhinolaryngology, Head } \\
\text { and Neck Surgery, } \\
\text { Inselspital, University } \\
\text { Hospital Bern and University } \\
\text { of Bern, Switzerland }\end{array}$ & $\begin{array}{l}\text { Data collection, database } \\
\text { setup, critical revision of the } \\
\text { article, final approval of the } \\
\text { version to be published }\end{array}$ \\
\hline $\begin{array}{l}\text { Athanasia } \\
\text { Korda, MD }\end{array}$ & $\begin{array}{l}\text { Department of } \\
\text { Otorhinolaryngology, Head } \\
\text { and Neck Surgery, } \\
\text { Inselspital, University } \\
\text { Hospital Bern and University } \\
\text { of Bern, Switzerland }\end{array}$ & $\begin{array}{l}\text { Data collection, database } \\
\text { setup, critical revision of the } \\
\text { article, final approval of the } \\
\text { version to be published }\end{array}$ \\
\hline $\begin{array}{l}\text { Franca } \\
\text { Wagner, MD }\end{array}$ & $\begin{array}{l}\text { University Institute of } \\
\text { Diagnostic and Interventional } \\
\text { Neuroradiology, Inselspital, } \\
\text { University Hospital Bern and } \\
\text { University of Bern, } \\
\text { Switzerland }\end{array}$ & $\begin{array}{l}\text { Data imaging analysis, } \\
\text { critical revision of the article, } \\
\text { final approval of the version } \\
\text { to be published }\end{array}$ \\
\hline
\end{tabular}

Thomas C. Department of Emergency Patient recruitment, critical
Sauter, MD Medicine, Inselspital, revision of the article, final University Hospital Bern approval of the version to and University of Bern, be published Switzerland

\begin{tabular}{lll}
\hline Hassen & Department of Neurology, & Data collection, critical \\
Kerkeni, MD & Inselspital, University & $\begin{array}{l}\text { revision of the article, final } \\
\text { approval of the version to }\end{array}$ \\
& $\begin{array}{l}\text { Hospital Bern and } \\
\text { University of Bern, } \\
\text { Switzerland }\end{array}$ & be published
\end{tabular}

\begin{tabular}{lll}
\hline $\begin{array}{l}\text { Roger Kalla, } \\
\text { MD }\end{array}$ & $\begin{array}{l}\text { Department of Neurology, } \\
\text { Inselspital, University } \\
\text { Hospital Bern and } \\
\text { University of Bern, } \\
\text { Switzerland }\end{array}$ & $\begin{array}{l}\text { Conceptualization, critical } \\
\text { revision of the article, final } \\
\text { approval of the version to } \\
\text { be published }\end{array}$ \\
\hline $\begin{array}{l}\text { Miranda } \\
\text { Morrison, MD }\end{array}$ & $\begin{array}{l}\text { Department of } \\
\text { Otorhinolaryngology, Head } \\
\text { and Neck Surgery, } \\
\text { Inselspital, University } \\
\text { Hospital Bern and University } \\
\text { of Bern, Switzerland }\end{array}$ & $\begin{array}{l}\text { Data analysis, critical } \\
\text { revision of the article, final } \\
\text { approval of the version to } \\
\text { be published }\end{array}$ \\
&
\end{tabular}

\begin{tabular}{lll}
\hline Marco & Department of & $\begin{array}{l}\text { Supervision, funding } \\
\text { Domenico }\end{array}$ \\
$\begin{array}{l}\text { Otorhinolaryngology, Head } \\
\text { Caversaccio, }\end{array}$ & $\begin{array}{l}\text { and Neck Surgery, } \\
\text { Inselspital, University }\end{array}$ & $\begin{array}{l}\text { of the article, final approval } \\
\text { of the version to be }\end{array}$ \\
MD & $\begin{array}{l}\text { Hospital Bern and University } \\
\text { published }\end{array}$ \\
& of Bern, Switzerland & \\
\hline
\end{tabular}

\section{References}

1. Hotson JR, Baloh RW. Acute vestibular syndrome. N Engl J Med. 1998;339(10): 680-685.

2. Kim HA, Yi HA, Lee H. Failure of fixation suppression of spontaneous nystagmus in cerebellar infarction: frequency, pattern, and a possible structure. Cerebellum. 2016; 15(2):182-189.

3. Lee H, Kim HA. Nystagmus in SCA territory cerebellar infarction: pattern and a possible mechanism. J Neurol Neurosurg Psychiatry. 2013;84(4):446-451. 
4. Newman-Toker DE, Kattah JC, Alvernia JE, Wang DZ. Normal head impulse test differentiates acute cerebellar strokes from vestibular neuritis. Neurology. 2008;70(24 pt 2):2378-2385.

5. Kattah JC, Talkad AV, Wang DZ, Hsieh YH, Newman-Toker DE. HINTS to diagnose stroke in the acute vestibular syndrome: three-step bedside oculomotor examination more sensitive than early MRI diffusion-weighted imaging. Stroke. 2009;40(11):3504-3510.

6. Kuruvilla A, Bhattacharya P, Rajamani K, Chaturvedi S. Factors associated with misdiagnosis of acute stroke in young adults. J Stroke Cerebrovasc Dis. 2011;20(6):523-527.

7. Grewal K, Austin PC, Kapral MK, Lu H, Atzema CL. Missed strokes using computed tomography imaging in patients with vertigo: population-based cohort study. Stroke. 2015;46(1):108-113.

8. Cnyrim CD, Newman-Toker D, Karch C, Brandt T, Strupp M. Bedside differentiation of vestibular neuritis from central "vestibular pseudoneuritis." J Neurol Neurosurg Psychiatry. 2008;79(4):458-460.

9. RobertBaloh WMF, Vicente Honrubia MDD, Kevin A, Kerber MD. Baloh and Honrubia's Clinical Neurophysiology of the Vestibular System. Oxford University Press; 2010.

10. Tarnutzer AA, Berkowitz AL, Robinson KA, Hsieh YH, Newman-Toker DE. Does my dizzy patient have a stroke? A systematic review of bedside diagnosis in acute vestibular syndrome. CMAJ. 2011;183(1):E571-E592.

11. Newman-Toker DE, Saber Tehrani AS, Mantokoudis G, et al. Quantitative videooculography to help diagnose stroke in acute vertigo and dizziness: toward an ECG for the eyes. Stroke. 2013;44(4):1158-1161.

12. Glasauer S, Kalla R, Büttner U, Strupp M, Brandt T. 4-aminopyridine restores visual ocular motor function in upbeat nystagmus. J Neurol Neurosurg Psychiatry. 2005; 76(3):451-453.

13. Tarnutzer AA, Weber KP, Schuknecht B, Straumann D, Marti S, Bertolini G. Gaze holding deficits discriminate early from late onset cerebellar degeneration. J Neurol. 2015;262(8):1837-1849.

14. Halmagyi GM, McGarvie LA, Strupp M. Nystagmus goggles: how to use them, what you find and what it means. Pract Neurol. 2020;20(6):446-450.

15. Schuchman G, Uri N. The variability of fixation suppression of caloric-induced nystagmus. J Laryngol Otol. 1986;100(7):765-768.
16. Baloh RW, Konrad HR, Honrubia V. Vestibulo-ocular function in patients with cerebellar atrophy. Neurology. 1975;25(2):160-168.

17. Kirkham TH, Guitton D, Katsarkas A, Kline LB, Andermann E. Oculomotor abnormalities in Friedreich's ataxia. Can J Neurol Sci. 1979;6(2):167-172.

18. Troost BT, Daroff RB. The ocular motor defects in progressive supranuclear palsy. Ann Neurol. 1977;2(5):397-403.

19. Takemori S, Aiba T, Shizawa R. Visual suppression of caloric nystagmus in brain-stem lesions. Ann NY Acad Sci. 1981;374:846-854.

20. Takemori S, Cohen B. Loss of visual suppression of vestibular nystagmus after flocculus lesions. Brain Res. 1974;72(2):213-224.

21. Leigh RJ, Zee DS. The Neurology of Eye Movements. Oxford University Press; 2015.

22. West PDB, Sheppard ZA, King EV. Comparison of techniques for identification of peripheral vestibular nystagmus. J Laryngol Otol. 2012;126(12):1209-1215.

23. Mantokoudis G, Tehrani AS, Wozniak A, et al. VOR gain by head impulse videooculography differentiates acute vestibular neuritis from stroke. Otology Neurotol. 2015;36(3):457-465

24. Hirvonen TP, Juhola M, Aalto H. Suppression of spontaneous nystagmus during different visual fixation conditions. Eur Arch Otorhinolaryngol. 2012;269(7): 1759-1762.

25. Watabe H, Hashiba M, Baba S. Voluntary suppression of caloric nystagmus under fixation of imaginary or after-image target. Acta Otolaryngol Suppl. 1996;525:155-157.

26. Ransohoff DF, Feinstein AR. Problems of spectrum and bias in evaluating the efficacy of diagnostic tests. N Engl J Med. 1978;299(17):926-930.

27. Chen L, Halmagyi GM. Video head impulse testing: from bench to bedside. Semin Neurol. 2020;40(1):5-17.

28. Korda A, Sauter TC, Caversaccio MD, Mantokoudis G. Quantifying a learning curve for video head impulse test: pitfalls and pearls. Front Neurol. 2020;11:615651.

29. Mantokoudis G, Saber Tehrani AS, Wozniak A, et al. Impact of artifacts on VOR gain measures by video-oculography in the acute vestibular syndrome. J Vestib Res. 2016, 26(4):375-385.

30. Mantokoudis G, Saber Tehrani AS, Kattah JC, et al. Quantifying the vestibulo-ocular reflex with video-oculography: nature and frequency of artifacts. Audiol Neurootol. 2015;20(1):39-50. 


\section{Neurology}

\section{Stroke Prediction Based on the Spontaneous Nystagmus Suppression Test in Dizzy \\ Patients: A Diagnostic Accuracy Study \\ Georgios Mantokoudis, Thomas Wyss, Ewa Zamaro, et al.}

Neurology 2021;97;e42-e51 Published Online before print May 13, 2021

DOI 10.1212/WNL.0000000000012176

\section{This information is current as of May 13, 2021}

\section{Updated Information \&} Services

References

Subspecialty Collections

Permissions \& Licensing

Reprints including high resolution figures, can be found at: http://n.neurology.org/content/97/1/e42.full

This article cites 28 articles, 10 of which you can access for free at: http://n.neurology.org/content/97/1/e42.full\#ref-list-1

This article, along with others on similar topics, appears in the following collection(s):

All Cerebrovascular disease/Stroke

http://n.neurology.org/cgi/collection/all_cerebrovascular_disease_strok e

All Neurotology

http://n.neurology.org/cgi/collection/all_neurotology

Class II

http://n.neurology.org/cgi/collection/class_ii

Nystagmus

http://n.neurology.org/cgi/collection/nystagmus

Vertigo

http://n.neurology.org/cgi/collection/vertigo

Information about reproducing this article in parts (figures,tables) or in its entirety can be found online at:

http://www.neurology.org/about/about_the_journal\#permissions

Information about ordering reprints can be found online:

http://n.neurology.org/subscribers/advertise

Neurology ${ }^{\circledR}$ is the official journal of the American Academy of Neurology. Published continuously since 1951, it is now a weekly with 48 issues per year. Copyright Copyright (C) 2021 The Author(s). Published by Wolters Kluwer Health, Inc. on behalf of the American Academy of Neurology.. All rights reserved. Print ISSN: 0028-3878. Online ISSN: 1526-632X.

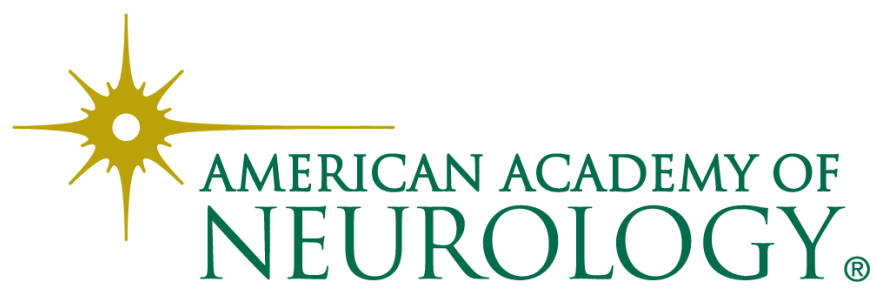

